\title{
Quark Matter in the Chiral Color Dielectric Model
}

\author{
Alessandro Drago ${ }^{a}$, Manuel Fiolhais ${ }^{b}$ and Ubaldo Tambini ${ }^{a}$ \\ ${ }^{a}$ Dipartimento di Fisica, Università di Ferrara, and INFN, Sezione di Ferrara, \\ Via Paradiso 12, Ferrara, Italy 44100 \\ ${ }^{b}$ Departamento de Física da Universidade \\ and Centro de Fisica Teórica, P-3000 Coimbra, Portugal
}

(August 23, 2019)

\begin{abstract}
We study the equation of state (EOS) of quark matter at zero temperature, using the Color Dielectric Model (CDM) to describe confinement. Sensible results are obtained in the version of the CDM for which confinement is imposed smoothly. The two-phases version of the model turns out to give unrealistic results for the EOS. Chiral symmetry plays a marginal rôle and the quarks are massive till high densities. The deconfinement phase transition is smooth and unlikely to be first order. Instabilities of the quark matter and the gap equation are discussed.
\end{abstract}

24.10.Jv Relativistic models,

24.85.+p Quarks, gluons and QCD in nuclei and nuclear processes

Typeset using REVTEX 


\section{INTRODUCTION}

The study of the Equation Of State (EOS) of Quark Matter (QM) has become a fashionable topic in view of the next experiments using heavy ions in program at RHIC(BNL) and at $\mathrm{LHC}(\mathrm{CERN})$ [1]. Furthermore the inner structure of neutron stars is now under investigation: the connection between the composition of the star and the cooling time, which can be measured, allows to discriminate among the various models, indicating the possible existence of a quark matter phase (see, e.g. [2]). The study of the equation of state of matter at high densities can also give usefull informations to traditional nuclear physics, since one can search heavy nuclei for precursor phenomena, both of the deconfinement and/or of the chiral restoration phase transition (for a review see [3]).

In many model calculations of the deconfinement phase transition the frame of the MIT bag model has been used [ [, 5]. In such a way a first order deconfinement phase transition is obtained (apart from specific, ad hoc choices of the model parameters) and the deconfinement phase transition coincide with the chiral restauration one. At densities and temperatures slightly bigger than the critical ones the right degrees of freedom are already quarks, having current masses, and perturbative gluons. There are anyway indications, from lattice calculations, that at temperatures bigger than the critical one non perturbative effects are still present in the quark-gluon plasma [6].

In this paper we study the EOS of QM using the Color Dielectric Model (CDM) to describe confinement [7]. We shortly review CDM in sect. 2. This model has been widely used to study both the static and the dynamical properties of the nucleon. Morover it can be used to describe many-nucleon systems: for a two nucleon system it allows to compute a nucleon-nucleon potential qualitatively similar to the ones used in nuclear physics [8]; in the case of a homogeneous, infinite system of nucleons the CDM can be used to construct a nonlinear version of the Walecka model [9].

The aim of our work is to extend previous calculations of the deconfinement phase transition, where the same model has been used [11. An important point of our calculation will be to fix the model parameters in order to reproduce the basic static properties of the single nucleon, as was already done in the study of the nucleon structure functions [12. We will later use the same parameters to study the EOS of QM, which we define as a system of totally deconfined quarks. In such a way the study of the QM's EOS will turn out to be a severe test for the different versions of the CDM, and we will be able to make some predictions of the properties of matter at high densities. Within CDM (with a double minimum potential for the scalar field), we will investigate the possibility of getting a scenario similar to the one described by the MIT bag model, with two phases undergoing a sharp first order phase transition (sect. 3). Our results show that such a description is incompatible with the CDM. We than study another version of the CDM (with a single minimum potential for the scalar field), where confinement is imposed more smoothly, and we get a sensible EOS for QM, without a sharp deconfinement transition.

An important feature of this deconfined quark matter is that quark's masses are big till high densities. Chiral restauration and deconfinement do not occur at the same density. The reasons why chiral simmetry is restaured so slowly are discussed in sect. 4 .

In the last sections we analyze the properties of QM, as described in the CDM. In sect. 5 the stability of quark matter is studied, using the technology of the response function. In 
sect. 6 we consider a gap equation, trying to understand the formation of quarks' clusters. Finally sect. 7 is devoted to the concluding remarks.

\section{THE COLOR DIELECTRIC MODEL}

\section{A. The model lagrangian}

In this section we shortly summarize the main features of the CDM. For a comprehensive review see the ones by Pirner [7] and by Birse [10].

We will use a chiral invariant version of the CDM, as the one used in Ref. [11 and also in Ref. [12. The Lagrangian reads

$$
\begin{aligned}
\mathcal{L} & =i \bar{\psi} \gamma^{\mu} \partial_{\mu} \psi+\frac{g}{\chi} \bar{\psi}\left(\sigma+i \gamma_{5} \vec{\tau} \cdot \vec{\pi}\right) \psi \\
& +\frac{1}{2}\left(\partial_{\mu} \chi\right)^{2}-U(\chi)+\frac{1}{2}\left(\partial_{\mu} \sigma\right)^{2}+\frac{1}{2}\left(\partial_{\mu} \vec{\pi}\right)^{2}-U(\sigma, \vec{\pi})
\end{aligned}
$$

where $U(\sigma, \vec{\pi})$ is the usual mexican-hat potential, as in ref. [15. $\mathcal{L}$ describes a system of interacting quarks, pions, sigmas and a scalar-isoscalar chiral singlet field $\chi$, whose potential $U(\chi)$ has an absolute minimum for $\chi=0$. In such a way, in the case of the single nucleon problem, the quarks' effective mass $-g \sigma / \chi$ diverges outside the nucleon. The simplest potential for $\chi$ is a quadratic one:

$$
U(\chi)=\frac{1}{2} M^{2} \chi^{2}
$$

Using this potential, $\chi$ fluctuates around zero, reaching this value asymptotically, at large distances. In the following we will refer to this version of the model as the Single Minimum (SM) one. Another possibility is to use a potential for $\chi$ having a Double Minimum (DM):

$$
U(\chi)=\frac{1}{2} M^{2} \chi^{2}\left[1+\left(\frac{8 \eta^{4}}{\gamma^{2}}-2\right) \frac{\chi}{\gamma M}+\left(1-\frac{6 \eta^{4}}{\gamma^{2}}\right)\left(\frac{\chi}{\gamma M}\right)^{2}\right]
$$

where the absolute minimum is still in the origin and the relative one is in $\chi=\gamma M$. Choosing appropriately the parameters it is possible to obtain solutions for the single nucleon where the $\chi$ field interpolates between the relative minimum at the center of the nucleon and the absolute minimum at big distances. In the following, we will consider for DM only solutions where $\chi$ has the above discussed behaviour. A complete analysis of the various possible solutions of the model (in a non-chiral-invariant version) can be found in Ref. 13.

In Fig.11 we show two typical solutions of the model for the single nucleon problem. As it appears, in the DM case the transition between the interior of the nucleon and the external region is sharper than in the SM, where all the fields have a very smooth behaviour. Correspondingly, the kinetic energy contribution (which comes mainly from the quarks) will be bigger in DM than in SM. This point will be relevant when studying the EOS of QM.

The Lagrangian is chiral invariant, and it can be considered a confining version of the traditional $\sigma$-model. An important point concern the value of the chiral fields, the pion and the sigma, in this model, in the single nucleon case. These fields are always near their 
vacuum value. This point has been checked out numerically several times and some euristic explanations have been proposed [10,14]. As a consequence, the chiral fields cannot 'wind' around the mexican hat potential. The pion is thus just a perturbation and cannot develop a non-trivial topology.

\section{B. Fixing the parameters}

The parameters of the model are: the chiral meson masses $m_{\pi}=0.14 \mathrm{GeV}, m_{\sigma}=1.2$ $\mathrm{GeV}$, the pion decay constant $f_{\pi}=0.093 \mathrm{GeV}$, the coupling constant $g$, and the parameters appearing in the quadratic (2) or in quartic $\chi$-potential (3).

The free parameters are fixed to reproduce the basic properties of the nucleon. At the mean field level we use the hedgehog ansatz which is an eigenstate of the so called Grand Spin $\vec{G}=\vec{S}+\vec{I}$, and is a superposition of various bare nucleon and delta states.

In order to describe the single nucleon state we performed a double projection on linear and angular momentum eigenstates from the hedgehog, whose details can be found in Ref. [15].

For the DM version of the model we use the sets of parameters of Ref. [16], for which a good description of the static properties of the nucleon was obtained.

For the SM version such a set of parameters was not available in the literature. We fixed the parameters $g$ and $M$ to reproduce the experimental value of the average mass of the nucleon and of the delta, and the isoscalar radius of the nucleon. Choosing $g=0.02$ $\mathrm{GeV}$ and $M=1.7 \mathrm{GeV}$ we got: $\left(E_{N}+E_{\Delta}\right) / 2=1.112 \mathrm{GeV}$ (exp.val. $=1.085 \mathrm{GeV}$ ) and $\left\langle r_{N}^{2}>_{i s o s c a l a r}^{1 / 2}=0.82 \mathrm{fm}(\right.$ exp.val. $=0.79 \mathrm{fm})$. These values depend essentially only on the quantity $G=\sqrt{g M}$. A detalied presentation of the single-nucleon properties in SM version of the CDM will be presented elsewhere [17].

To perform an exhaustive analysis of the various versions of the CDM, we considered also the possibility of having an effective mass term for the quarks in which the $\chi$ field appears with a power different from one: $m_{q}=-g^{p} \sigma / \chi^{p}$. Studying this possibility in the SM version, and for $p=2$, a good description of the single nucleon properties can be achieved using $g=0.02 \mathrm{GeV}$ and $M=1.10 \mathrm{GeV}\left(\left(E_{N}+E_{\Delta}\right) / 2=1.102 \mathrm{GeV}\right.$ and $\left\langle r_{N}^{2}>_{\text {isoscalar }}^{1 / 2}=0.78 \mathrm{fm}\right)$.

Several important differences exist between the CDM and the MIT bag model. In the latter model, inside the bag the quarks have current masses of few $\mathrm{MeV}$. The bag is stabilized through the introduction of a big vacuum pressure, of the order of $150 \mathrm{MeV} / \mathrm{fm}^{3}$. Perturbative gluons are considered to be the right degrees of freedom inside the bag. In the CDM model, in all versions, the effective quark mass is everywhere bigger than a number of the order of $100 \mathrm{MeV}$, hence chiral symmetry is broken and Goldstone bosons are the right degrees of freedom. We will come back later to this point, comparing the EOS of QM as computed in the chiral CDM with the one computed in a non-chiral version [18]. In the CDM a vacuum pressure is also present, coming from the $\chi$ field: this pressure is roughly constant inside the nucleon in the DM case, where it equals $U(\chi=\gamma M)=M^{4} \eta^{4}$, whilst in the SM case the pressure $\frac{1}{2} M^{2} \chi(r)^{2}$ depends on $r$. It is important to stress that in the DM version of the $\mathrm{CDM}$ model the pressure is very small, of the order of few $\mathrm{MeV}$, and this point will also be important when discussing the EOS of QM. 


\section{MEAN FIELD APPROXIMATION TO THE EOS OF QM}

In this section we will study the EOS of QM, using the Lagrangian of the chiral CDM $(1-3)$.

For QM we mean a system of totally deconfined quarks, described using plane waves, with the $\chi$, pion and sigma fields having a constant value, given by the Euler-Lagrange equations.

The total energy of QM in the mean field approximation is the following:

$$
E_{Q M}=12 V \int \frac{d \mathbf{k}}{(2 \pi)^{3}} \sqrt{\mathbf{k}^{2}+\left(\frac{g \bar{\sigma}}{\bar{\chi}}\right)^{2}} \theta\left(k_{F}-k\right)+V U(\bar{\chi})+V U(\bar{\sigma}, \vec{\pi}=0),
$$

where $k_{F}$ is the Fermi momentum of quarks, $\bar{\chi}$ and $\bar{\sigma}$ are the solutions of the coupled equations

$$
\begin{gathered}
\left.\frac{d U(\chi)}{d \chi}\right|_{\chi=\bar{\chi}}=-g \bar{\sigma} \frac{\rho_{S}(\bar{\chi}, \bar{\sigma})}{\bar{\chi}^{2}}, \\
\left.\frac{d U(\sigma, \vec{\pi}=0)}{d \sigma}\right|_{\sigma=\bar{\sigma}}=-g \frac{\rho_{S}(\bar{\chi}, \bar{\sigma})}{\bar{\chi}},
\end{gathered}
$$

and the scalar density $\rho_{S}(\bar{\chi}, \bar{\sigma})$ is given by

$$
\rho_{S}(\bar{\chi}, \bar{\sigma})=<\bar{\psi} \psi>=12 \int \frac{d \mathbf{k}}{(2 \pi)^{3}} \frac{g \bar{\sigma} / \bar{\chi}}{\sqrt{\mathbf{k}^{2}+(g \bar{\sigma} / \bar{\chi})^{2}}} \theta\left(k_{F}-k\right) .
$$

In the mean field approximation, for an homogeneous infinite system, $\langle\vec{\pi}\rangle=0$, so the pionic field is not contributing (of course it indirectly enters the EOS, because our model parameters are fixed in the single nucleon problem, where $<\vec{\pi}>\neq 0$ ). This is drawback of the mean field approximation, when applied to homogeneous infinite systems. A way to circumvent this problem is discussed by Ghosh and Phatak [19].

It is important to analyze the behaviour of the $\chi$ field, both in the SM and in the DM version of the model, when the CDM is used to describe a collection of nucleons at increasing densities. First of all, in the DM model a critical density exist, for which the $\chi$ field undergo a discontinous jump. In the appendix a proof of this statement is given, based on the study of a two-nucleon system for various internucleon distances. For distances smaller than a critical one, the $\chi$ field, in the region between the two nucleon, will cease to interpolate between the two minima, as discussed in Sec.2B, and will stay near the relative minimum. This transition cannot be made continuous. In the DM version of the CDM, the deconfinement phase transition is therefore a discontinous first order transition. In the SM version of the model nothing similar can happen. Of course this is not enaugh to conclude that, in this case, the deconfinement phase transition is not first order. We will study more in detail what happens in Sec.5 and 6.

We will now compare the EOS of QM, as computed in our model, with the EOS of nuclear matter as obtained in the Walecka model [20]. In Fig.2] our results for the EOS are shown.

In the DM case we used the parameter sets 15,16]: 


$$
\begin{aligned}
& \text { i): } g=0.059 \mathrm{GeV}, M=1.4 \mathrm{GeV}, \gamma=0.04, \eta=0.06 \\
& \text { ii) } g=0.029 \mathrm{GeV}, M=1.2 \mathrm{GeV}, \gamma=0.06, \eta=0.06 \\
& \text { iii): } g=0.0235 \mathrm{GeV}, M=1.6 \mathrm{GeV}, \gamma=0.03, \eta=0.06
\end{aligned}
$$

As it appears, the energy per baryon number in DM is very small, its value being below the one given by the Walecka model for almost all densities. The DM version of the CDM is therefore unrealistic when used to describe the EOS of QM. A similar result was obtained in a calculation performed in a non-chiral version of CDM [18]. The reasons for such a débâcle are to be find in the very small value of the pressure in DM. If one would add 'by hand' a pressure's contribution of the order of $150 \mathrm{MeV} / \rho$ to the energy per baryon shown in Fig.2 for DM, one will get a result similar to the one obtained using the MIT bag model. The problem is that such a big pressure cannot be obtained in the CDM, because corresponds to a solution for the single nucleon problem where the quark fields are very steep: as it has been shown by Leech and Birse [21], the center of mass motion cannot be projected out consistently in this case, and the mean field approximation is no more a good starting point.

In the SM with $p=1$ case (see Sect.2B), on the other hand, the mean field approximation to the EOS of QM gives a sensible result: the energy per baryon number is bigger in the QM phase than in the hadronic phase for all densities smaller or of the order of $\rho_{e q}=0.17 \mathrm{~N} / \mathrm{fm}^{3}$, the equilibrium density of nuclear matter. After this density the equation of state of QM and the one of nuclear matter seems almost equivalent, till densities of the order of $2 \rho_{e q}$, after which the energy of QM is smaller than the energy of nuclear matter. We would like to remind that we have not modified the parameters of the model, but we are sticking to the ones fixed to the static properties of the nucleon, as discussed in Sec.2.

The last possibility we have considered is SM with $p=2$. Also in this case, the EOS of quark matter that one obtains after fixing the parameters is too low, as it can be seen from Fig.2.

We can conclude from analysis of all the versions of the CDM, that the most realistic EOS of the quark matter is obtained using the model in which confinement is imposed in the smoothest way, i.e. SM with $p=1$. In the following sections, only this version will be considered.

The most relevant feature of the result for $\operatorname{SM} p=1$, is the wide range of densities for which the EOS of traditional nuclear matter and the EOS of quark matter are almost equivalent. The difference in energy between the two phases is of some tens' $\mathrm{MeV}$, only. It is remarkable that this 'almost equivalence' starts at a density of the order of $\rho_{e q}$. A natural interpretation of this result is that in the case of heavy nuclei, some precursor phenomena of deconfinement could be seen (as swelling, for instance), but no dramatic change is going to happen in the system till much higher densities. In Sec.6 we will analyze the possibility that the small energy gap between the two phases can be explained taking into account correlations in QM, using Bethe-Goldstone equation.

\section{QUARKS' MASS AND CHIRAL SYMMETRY RESTAURATION}

We discuss now more in details the dependence of quarks' effective mass $m_{q}=-g \sigma / \chi$ on the density. In this model two different mechanisms are at work to reduce $m_{q}$ : one comes 
from the chiral field sigma, that moves from its vacuum value $-f_{\pi}$ as the density increases (in the following we will not take into account a chiral-symmetry breaking term giving mass to the pion, because it is irrelevant to what we want to discuss). The other mechanism that modifies $m_{q}$ is confinement, through the $\chi$ field, which moves away from zero. As we will see, this second mechanism is the relevant one till high densities.

We restrict our discussion to the SM $p=1$ version of the model, the one which gives sensible results for the EOS of QM. In this case, eq.(5) can be formally solved, giving

$$
\bar{\chi}=\left(-\frac{g}{M^{2}} \bar{\sigma}<\bar{\psi} \psi>\right)^{1 / 3} .
$$

This is not really the solution of eq.(5), because the scalar density $\rho_{S}=\langle\bar{\psi} \psi>$ still depends on $\bar{\chi}$ through the fermions' mass. It can nevertheless be used as an approximation to the real solution, if one neglects the difference between $\rho=\left\langle\psi^{\dagger} \psi>\right.$ and $\rho_{S}$ (this approximation is not too bad, because, as we shall see, quarks' masses are not decreasing very fast).

The 'Maxican hat' potential, entering the Lagrangian (1), and parametrized as in Ref. |15, is

$$
U(\sigma, \pi)=\frac{m_{\sigma}^{2}}{8 f_{\pi}^{2}}\left(\sigma^{2}+\pi^{2}-f_{\pi}^{2}\right)^{2},
$$

where $m_{\sigma}$ is the mass of the sigma field. Using the formal solution for $\chi$ given in eq.(8), one can compute the shift $\Delta$ of the sigma field from its vacuum value

$$
\begin{gathered}
\bar{\sigma}=-f_{\pi}+\Delta \\
\Delta=\frac{1}{m_{\sigma}^{2}}\left[\frac{(g M)^{2}}{f_{\pi}}\right]^{1 / 3}(\bar{\psi} \psi)^{2 / 3} \equiv C(\bar{\psi} \psi)^{2 / 3} .
\end{gathered}
$$

Substituting in eq.(11) typical numbers, and in particular the ones we used in Sec.3, the dimensional coefficient $C$ in eq.(11) turns out to be small, of the order of $0.1 \mathrm{fm}$, and $\Delta$ is therefore also small, if compared to $f_{\pi}$, till very high densities.

In Fig. 3 we compare the effective quark mass, as computed taking into account both the reductions coming from the $\chi$ field and from the $\sigma$ field (solid line), with the effective mass when the $\sigma$ field is kept fixed at $-f_{\pi}$ (dashed line). At a density of order of $3 \rho_{e q}$, the effective mass of the quarks is reduced from its value at $\rho_{e q}$ by a factor $\simeq 0.8$. Comparing the two lines of Fig. 3 , one can see that most of the effect is due to the confining $\chi$ field.

\section{QUARK MATTER INSTABILITIES}

The more direct way to study the instabilities of a many-body system is to look where its compressibility $K$ becomes infinite. The compressibility is related to the pressure through the following relations

$$
P=\rho^{2} \frac{\partial(E / N)}{\partial \rho}
$$




$$
K^{-1}=\rho \frac{\partial P}{\partial \rho}
$$

Here $(E / N)$ is the energy per particle and $\rho$ is the density.

In Fig. 4 the compression modulus $K^{-1}$ is shown (here and in the following we will refer to the SM version of the CDM, only). As it can be seen, quark matter becomes unstable, in our calculation, at a density smaller than $\rho_{\text {inst }} \simeq \rho_{\text {eq }} / 2$. This instability is not due to the fact that in this region the energy per particle of quark matter is higher than that of nuclear matter, but it is related to the possibility of creating undamped density fluctuations in the system, without spending energy.

To clarify even more this point, one can try to reproduce the same result, studying the collective excitations of the system, which can be found searching for the poles of the propagators of the scalar fields, dressed by the particle-hole polarization propagator. In the following we will only discuss the collective states coming from the propagation of the $\chi$ and the sigma field, leaving the discussion of the pion propagator, and the related phenomena, to a future study.

To define the propagators of the $\chi$ and the sigma, we expand these fields around their mean field value:

$$
\begin{aligned}
& \sigma=\bar{\sigma}+\tilde{\sigma} \\
& \chi=\bar{\chi}+\tilde{\chi}
\end{aligned}
$$

The mass term reads thus $(<\vec{\pi}>=0)$

$$
g \frac{\bar{\psi} \sigma \psi}{\chi}=g \frac{\bar{\psi}(\bar{\sigma}+\tilde{\sigma}) \psi}{(\bar{\chi}+\tilde{\chi})}=g \frac{\bar{\psi} \bar{\sigma} \psi}{\bar{\chi}}+g \frac{\bar{\psi} \tilde{\sigma} \psi}{\bar{\chi}}-g \frac{\bar{\psi} \bar{\sigma} \psi}{\bar{\chi}^{2}} \tilde{\chi}+\ldots
$$

where we have expanded the $\chi$ field till first order in the fluctuation. Since we are looking for the instabilities of the system, i.e. for the situations in which an (arbitrary small) fluctuation around the mean field develops spontaneously and propagates undamped, it is enough to consider a first order expansion in $\tilde{\chi}$.

From the linearized lagrangian (16), we read the couplings between the scalar fields $\tilde{\chi}$ and $\tilde{\sigma}$ and the quark fields

$$
\begin{gathered}
g_{\tilde{\chi}}=-g \frac{\bar{\sigma}}{\bar{\chi}^{2}} \\
g_{\tilde{\sigma}}=\frac{g}{\bar{\chi}} .
\end{gathered}
$$

The masses corresponding to the $\tilde{\chi}$ and to the $\tilde{\sigma}$ fluctuations are given by the following relations

$$
M_{\tilde{\chi}}^{* 2} \equiv<\frac{\partial^{2}}{\partial \tilde{\chi}^{2}}\left[U(\chi)-g \frac{\bar{\psi} \sigma \psi}{\chi}\right]>=M^{2}-2 g \frac{\bar{\psi} \sigma \psi}{\chi^{3}}=3 M^{2}
$$




$$
m_{\tilde{\sigma}}^{* 2} \equiv<\frac{\partial^{2} U(\sigma, \vec{\pi})}{\partial \tilde{\sigma}^{2}}>
$$

In the previous equations, the brakets $<>$ indicate mean value in the mean field approximation, where the fluctuations are set equal to zero. In eq.(19), we have used the field equation for $\bar{\chi}(5)$, to eliminate the dependence on the fields. The mass of the $\tilde{\sigma}$ is only slightly reduced from its value at zero-density, because of the slowness of the chiral symmetry restauration (see the discussion in the previous section).

The mean field propagators of the scalar fields are thus

$$
\begin{gathered}
D_{0}^{\sigma}\left(q_{\mu}\right)=1 /\left(q_{\mu}^{2}-m_{\tilde{\sigma}}^{* 2}+i \eta\right) \\
D_{0}^{\chi}\left(q_{\mu}\right)=1 /\left(q_{\mu}^{2}-M_{\tilde{\chi}}^{* 2}+i \eta\right) .
\end{gathered}
$$

We define the scalar polarization propagator in the following way

$$
\Pi_{s}(q)=-i \int \frac{d^{4} k}{(2 \pi)^{4}} \operatorname{Tr}[G(k) G(k+q)]
$$

where $G(k)$ is the single quark relativistic propagator in the medium which can be decomposed in the Feynman propagator and a density dependent correction

$$
G(k)=\left(\gamma^{\mu} k_{\mu}+m_{q}\right)\left[\frac{1}{k_{\mu}^{2}-m_{q}^{2}+i \eta}+\frac{i \pi}{E^{*}(k)} \delta\left(k_{0}-E^{*}(k)\right) \theta\left(k_{F}-|\vec{k}|\right)\right] \equiv G_{F}(k)+G_{D}(k) .
$$

The effective quark mass is computed from mean field equations: $m_{q}=-g \bar{\sigma} / \bar{\chi}$, and $E^{*}(k)=$ $\sqrt{m_{q}^{2}+k^{2}}$. For a review of the formalism, see Reff. [20,22].

In accordance with the mean field approximation, we neglect the vacuum fluctuations effects, and we take only the density-dependent part of the polarization propagator [22]. In such a way we avoid possible complications coming from the ill-defined zero density vacuum state. An explicit expression for $\Pi_{s}$ can be found in Ref. [22].

We now consider the propagators of the scalar fields, modified by the polarization propagator insertion, i.e. the propagators in the RPA approximation. The $\tilde{\chi}$ and the $\tilde{\sigma}$ propagators mix up in the RPA approximation. They are the solution of the following coupled equations

$$
\begin{aligned}
& D^{\sigma \sigma}=D_{0}^{\sigma}+D_{0}^{\sigma} g_{\tilde{\sigma}} \Pi_{s}\left(g_{\tilde{\sigma}} D^{\sigma \sigma}+g_{\tilde{\chi}} D^{\chi \sigma}\right) \\
& D^{\chi \chi}=D_{0}^{\chi}+D_{0}^{\chi} g_{\tilde{\chi}} \Pi_{s}\left(g_{\tilde{\chi}} D^{\chi \chi}+g_{\tilde{\sigma}} D^{\sigma \chi}\right) \\
& D^{\chi \sigma}=D_{0}^{\chi} g_{\tilde{\chi}} \Pi_{s}\left(g_{\tilde{\sigma}} D^{\sigma \sigma}+g_{\tilde{\chi}} D^{\chi \sigma}\right) \\
& D^{\sigma \chi}=D_{0}^{\sigma} g_{\tilde{\sigma}} \Pi_{s}\left(g_{\tilde{\chi}} D^{\chi \chi}+g_{\tilde{\sigma}} D^{\sigma \chi}\right) .
\end{aligned}
$$

In the previous equation, the dressed propagators correspond to various situations in which the two mean field propagators $D_{0}^{\sigma}$ and $D_{0}^{\chi}$ mix among themselves via the polarization propagator. These propagators can be decoupled, giving the following RPA propagators 


$$
\begin{aligned}
D^{\sigma \sigma} & =D_{0}^{\sigma}\left(1-g_{\tilde{\chi}} \Pi_{s} g_{\tilde{\chi}} D_{0}^{\chi}\right) / \epsilon \\
D^{\chi \chi} & =D_{0}^{\chi}\left(1-g_{\tilde{\sigma}} \Pi_{s} g_{\tilde{\sigma}} D_{0}^{\sigma}\right) / \epsilon \\
D^{\chi \sigma} & =D_{0}^{\chi} g_{\tilde{\chi}} \Pi_{s} g_{\tilde{\sigma}} D_{0}^{\sigma} / \epsilon \\
D^{\sigma \chi} & =D_{0}^{\sigma} g_{\tilde{\sigma}} \Pi_{s} g_{\tilde{\chi}} D_{0} \chi / \epsilon \\
\epsilon & =1-\left(g_{\tilde{\chi}} \Pi_{s} g_{\tilde{\chi}} D_{0}^{\chi}+g_{\tilde{\sigma}} \Pi_{s} g_{\tilde{\sigma}} D_{0}^{\sigma}\right) .
\end{aligned}
$$

The collective excitations of the system correspond to the zeros of the denominator $\epsilon\left(q_{\mu}\right)$. Instabilities are the collective states at zero energy transferred [22], i.e. the solutions of the equation

$$
\epsilon\left(q_{0}=0, \vec{q}\right)=0
$$

The solutions of this equation are shown in Fig., as a function of the transferred momentum and of the density of the system. In the region enclosed by the line, corresponding to the solutions of eq. (27), the system is instable; in particular, when these instabilities exist for zero transferred momentum the system develops spontaneously undamped density fluctuations. The range of densities for which the system is found to be instable (at zero momentum transferred) coincides with the range found studying the compressibility.

Concerning the interplay between chiral symmetry and confinement in the development of the instabilities, we found again that chiral symmetry plays a marginal rôle in the model: if one takes into account the $\tilde{\chi}$ propagator only, the instability region shown in Fig. almost unchanged.

\section{TWO-BODY CORRELATIONS: GAP EQUATION}

We will now study two-body correlations, using the formalism of the gap equation [23]. The gap is the difference between the energy of the incorrelated pair and the energy of the correlated pair. It is thus positive if the potential is attractive. We will use as the residual interaction the one arising from the exchange of a $\tilde{\chi}$ or a $\tilde{\sigma}$. Of course we are able to take into account only two-body correlations, and three body-correlations can be even more important.

The gap equation reads [23]

$$
\Delta_{k}=\frac{1}{2} \sum_{\mathbf{k}^{\prime}}<\mathbf{k},-\mathbf{k}|V| \mathbf{k}^{\prime},-\mathbf{k}^{\prime}>\frac{\Delta_{k^{\prime}}}{\left(\Delta_{k^{\prime}}^{2}+\xi_{k^{\prime}}^{2}\right)^{1 / 2}}
$$

Here we are following the convenction [23] that an attractive potential is positive. The potential $V$ is evaluated between incorrelated states, described by plane waves. $\xi_{k}$ is the single-particle energy, mesured relative to the chemical potential $\mu=\sqrt{k_{F}^{2}+m_{q}^{2}}$. We will not use the relativistic reduction of $\xi_{k}$, because the relativistic corrections are not totally

negligible. Therefore $\xi_{k}=\sqrt{k^{2}+m_{q}^{2}}-\sqrt{k_{F}^{2}+m_{q}^{2}}$, where $m_{q}$ is the quark mass as computed in the mean field approximation.

As we have already said, the potential $V$ arises from the exchange of the scalar fields' fluctuations $\tilde{\chi}$ and $\tilde{\sigma}$. The masses of this fluctuations and the couplings between the fluctuations and the quarks' fields, have been obtained in the previous section, expanding the 
lagrangian around the mean field approximation. Of course this expansion, which was sufficient to study the instabilities of the system, is in general quite a crude approximation. The results obtained for the gap are thus only the first step in the study of the difficult problem of the clusterization.

The potential $V$ is a Yukawa potential, but an extra factor $m_{q}^{2} / E^{*}(k) E^{*}\left(k^{\prime}\right)$ has to be

included $\left(E *(k)=\sqrt{k^{2}+m_{q}^{2}}\right)$, because of the choosen normalization of the quarks' spinors.

Following Ref. [23], since the resulting gap $\Delta_{k}$ is much smaller than the Fermi energy, the integrand appearing in eq.(28) is sharply peaked near $\xi=0$, and then $\Delta_{k} \simeq \Delta_{k_{F}} \equiv \Delta$, i.e. the gap is almost independent on the momentum.

We show in Fig. 团 the resulting gap. It shows a strong dependence on the density, and for densities of the order of $\rho_{e q}$ is already totally negligible.

The gap equation can be solved analytically for small value of the gap. The result is

$$
\Delta \approx 8 \frac{k_{F}^{2}}{2 m_{q}} \exp \left(-\frac{\pi k_{F}^{2} / 2 m_{q}}{<k_{F}|V| \phi_{k_{F}}>}\right),
$$

where the matrix element of the potential is

$$
<k_{F}|V| \phi_{k_{F}}>\equiv k_{F} \int_{0}^{\infty} \sin k_{F} x V(x) \sin k_{F} x \mathrm{~d} x .
$$

Since our residual interaction $V$ is always attractive, it doesn't exist a critical density at which the gap is exactly zero, and this value is reached only asymptotically.

\section{CONCLUSIONS}

In this paper we have studied quark matter, using the non-perturbative tool of the CDM model. Let us summarize our main results:

- of all the considered versions of the model, only one gives sensible results, i.e. the one in which confinement is imposed in the smoothest way. The other versions of the model give an exceedingly low energy per baryon number for the quark matter.

- the SM $(p=1)$ version gives an EOS for the quark matter which is almost identical to the EOS of nuclear matter as computed using the Walecka model, for the range of densities $\rho_{e q} \leq \rho \leq 2 \rho_{e q}$. In this range, the difference in the energy per baryon number between the nuclear matter and the quark matter is very small. Taking into account the theoretical incertitude in the fixing of the parameters (Sec.2B), this energy difference is of the order of $20 \mathrm{MeV}$. At densities smaller than $\rho_{e q}$ the energy difference rapidly increases, and for densities higher than $2 \rho_{e q}$ the quark matter is the energetically most favourable state. An important point is that the minimum for the EOS of quark matter is at a density of the order of $\rho_{e q}$, and this result does not depend on the fine tuning of the parameters.

- the mass of the quarks remains big (of the order of $100 \mathrm{MeV}$ ) till high densities, much higher than the density at which quark matter becomes the ground state. The deconfinement phase transition and the chiral symmetry restauration arise at totally different densities.

- the quark matter (in SM and $\mathrm{p}=1$ ) becomes unstable at low densities, of the order of $\rho_{e q} / 2$. The instability can be obtained both from the study of the compressibility (where the compressibility becomes negative the system is unstable) and from the study of the collective states at zero energy transfer. The two method give the same critical density. 
- the process of clusterization can be studied considering the correlations between the particles, beyond the mean field approximation. The gap we obtained seems rather small. We have to bear in mind that we have oversimplified the problem, by linearizing the residual interaction (and thus getting an approximate propagator for $\tilde{\chi}$, good only for small fluctuations), and by considering only two body correlations, where the three body ones are probably the most relevant.

To conclude, we would like to consider three possible applications of the model.

- Cooling of neutron stars (see C.J.Pethick in [3]).

A mechanism called URCA has been invoked to explain the rapid cooling of neutron stars. This mechanism proceeds via the exchange of electrons between neutrons and protons, which cool down emitting neutrinos and antineutrinos:

$$
\begin{aligned}
& n \rightarrow p+e^{-}+\bar{\nu}_{e} \\
& p+e^{-} \rightarrow n+\nu_{e}
\end{aligned}
$$

A minimal fraction of protons is required, in order to fulfil momentum and energy conservation. This critical fraction is of the order of $1 / 9$. Using traditional nuclear physics models to compute the protons' fraction, one gets numbers slightly smaller than the critical one, and the URCA mechanism cannot start.

Another possibility is to invoke the presence of quark matter in the core of the star, and to consider reactions similar to the one previously described, but with the electron now exchanged between up and down quarks. In this case the problem is that, considering quark matter as described by the MIT bag model, quarks are massless and the phase space is thus zero. Therefore one would need a massive quark matter phase, and the possibility of reaching this phase at the density of the core of neutron stars, tipically of the order of $5 \rho_{e q}$. This situation is actually the one described by the $\mathrm{SM}(\mathrm{p}=1)$ version of the CDM. URCA mechanism should therefore be possible, and with an high luminosity, too.

- Energy released in supernova explosion.

Using a traditional nuclear physics approach, the energy released in supernova explosion is generally too small. A softer EOS could solve the problem, but if one uses e.g. the MIT bag to study matter at high density, the deconfinement phase transition is reached at densities larger than the one presumably reached in the collapse of the star. The EOS for matter at high density as computed in the CDM, is softer than the EOS of nuclear matter, and presumably a similar result will be obtained when computing neutron matter. Furthermore the softening starts at densities of the order of $2 \rho_{e q}$.

- EMC effect and swelling of the nucleon.

To conclude let us consider the problem of the possible swelling of the nucleons embedded in a nucleus. If one considers,e.g. electron-scattering on heavy nuclei, one realizes that the swelling is a sensible mechanism, but it must be of the order of some $5 \%$ in order to be realistic. The real problem is thus not the one to obtain a swelling, but to obtain a not too big effect. In other words, the nucleons have not to dissolve when embedded in a nucleus. Since the minimum of the EOS of the quark matter is for a density near $\rho_{e q}$, only in the center of heavy nuclei some swelling mechanism can appear. The exact amount of swelling depends on the precise difference in energy between the quark matter and the nuclear matter at densities $\sim \rho_{e q}$, and is beyond the possibility of the present calculation.

We are now carrying out researches in all the directions previuosly outlined. 


\section{APPENDIX:}

In this appendix we discuss the behaviour of the $\chi$ field in the DM version of the CDM, for a two nucleon system, as a function of their distance $d$, which is related to the density of the system $\rho \propto d^{-3}$. We will show that a critical internucleon distance exists, at which the $\chi$ field has a discontinuous behaviour.

For simplicity we will omit the chiral fields, the pion and the sigma, from our discussion, considering the following Lagrangian:

$$
\mathcal{L}=i \bar{\psi} \gamma^{\mu} \partial_{\mu} \psi-\frac{g}{\chi} \bar{\psi} f_{\pi} \psi+\frac{1}{2}\left(\partial_{\mu} \chi\right)^{2}-U(\chi)
$$

where the potential for $\chi$ is the one given in eq.(3) and shown in Fig.5a. We have labelled by $\chi_{m}$ the relative minimum and by $\chi_{1}$ the other value of $\chi$ for which $U\left(\chi_{1}\right)=U\left(\chi_{m}\right)=M^{4} \eta^{4}$.

We consider a system made of two clusters of three quarks each, with an intercluster distance $d$. In the mean field approximation the total energy of the two-nucleon system is given by

$$
E_{2}=6 \epsilon_{q}+T_{\chi}+E_{\chi}
$$

where $\epsilon_{q}$ is the energy of the single quark, $T_{\chi}$ is the energy of the $\chi$ field associated with its spatial fluctuations 1 and $E_{\chi}$ is the energy coming from the potential $U(\chi)$.

When the intercluster distance $d$ is large, the $\chi$ field interpolates between a number slightly bigger than $\chi_{m}$ and a small number, smaller than $\chi_{1}$, in the internucleon space (see Fig.5b, the solid and dotted line).

When the distance $d$ is reduced, the value of the scalar field in the internucleon region increases. We compare now two possible solutions: one in which the $\chi$ field has a minimum value in the internucleon region, equal to $\chi_{1}$, and a second in which the $\chi$ field remains almost constant in the internucleon region (solid and dashed line in Fig.5b). The second solution has a smaller energy than the first one. In fact: $\epsilon_{q}$ is smaller, because the quarks move in a single big well, instead of moving in a double well; $T_{\chi}$ is smaller because $\chi$ is fluctuating less; $E_{\chi}$ is smaller because $\chi$ is not moving (in the internucleon region) through the relative maximum of $U(\chi)$. Since the second solution has a smaller energy, the value of $\chi$ in the internucleon region will not smoothly increse, as the distance $d$ is decreased, but will jump, at a certain critical distance, from the behaviour described by the solid and dotted line in Fig.5b to the one described by the solid and dashed line. The internucleon value of $\chi$ can be assumed as an order parameter that undergo a discontinuous change as the density is increased, thus the transition is first order.

\footnotetext{
${ }^{1}$ This energy is not the kinetic energy of the $\chi$ field, which is zero, because $\chi$ is a scalar field and it is assumed to be time independent.
} 


\section{REFERENCES}

[1] Quark Matter '93, Nucl.Phys.A566(1994).

[2] Neutron Stars: Theory and Observation, J. Ventura and D.Pines eds. (Kluwer Acad. Pub., 1991).

[3] Realistic Nuclear Structure, G.Brown, P.Ellis, E.Osnes and D.Strottman eds. Phys.Rep.242(1994).

[4] J.W.Clark, J.Cleymans and J.Rafelski, Phys.Rev.C33(1986)703.

[5] R.Tamagaki and T.Tatsumi, Progr.Theor.Phys.Supp.112(1993)277.

[6] J.-P.Blaizot, Quark Matter '93, Nucl.Phys.A566(1994)333c.

[7] H.J.Pirner, Prog.Part.Nucl.Phys.29(1992)33.

[8] K.Bräuer, A.Drago and A.Faessler, Nucl.Phys.A511(1990)558.

[9] E.Naar and M.C.Birse, J.Phys.G:Nucl.Part.19(1993)555.

[10] M.C.Birse, Progr.Part.Nucl.Phys.25(1990)1

[11] W.Broniowski, M.Čibej, M.Kutschera and M.Rosina, Phys.Rev.D41(1990)285.

[12] V.Barone, A.Drago and M.Fiolhais, Phys.Lett.B338(1994)433.

[13] W.Broniowski, M.K.Banerjee and T.D.Cohen, Univ. of Maryland preprints ORO 5126298 (1986).

[14] A.Drago, K.Bräuer and A.Faessler, J.Phys.G15(1989)L7.

[15] T.Neuber, M.Fiolhais, K.Goeke and J.N.Urbano, Nucl.Phys.A560(1993)909.

[16] M.Fiolhais, T.Neuber, K.Goeke, P.Alberto and J.N.Urbano, Phys.Lett.B268(1991)1.

[17] V.Barone, A.Drago, M.Fiolhais and U.Tambini, work in progress.

[18] V.Barone and A.Drago, submitted for pubblication.

[19] S.K.Ghosh and S.C.Phatak, J.Phys.G18(1992)755.

[20] B.D.Serot and J.D.Walecka, Adv.Nucl.Phys.16(1986)1.

[21] R.C.Leech and M.C.Birse, Nucl.Phys.A494(1989)489.

[22] K.Lim and C.J.Horowitz, Nucl.Phys.A501(1989)729.

[23] A.L.Fetter and J.D.Walecka, Quantum Theory of Many-Particle Systems McGraw-Hill 1971. 


\section{FIGURES}
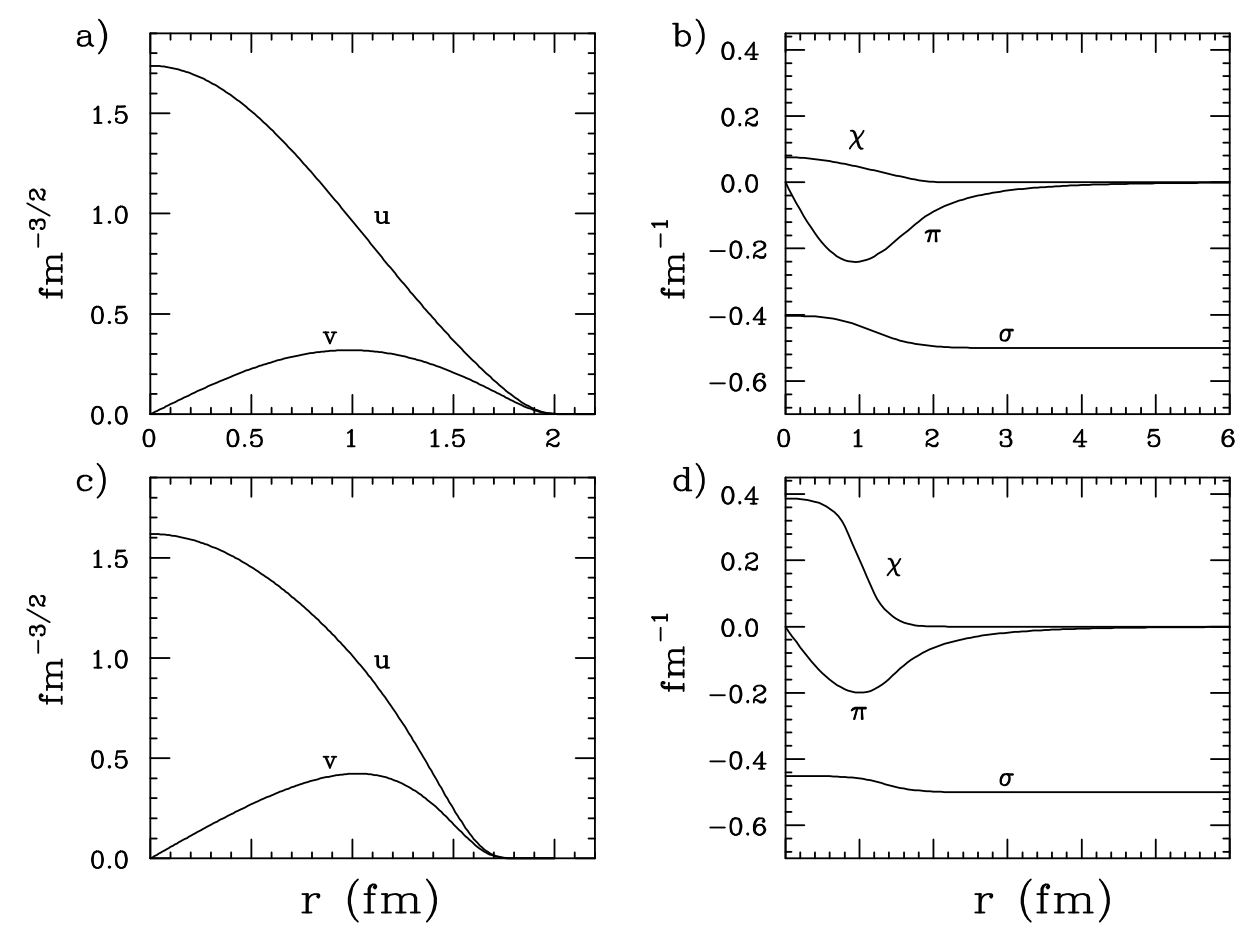

FIG. 1. Typical solutions of the model for single nucleon problem in the SM (a,b) and DM $(\mathrm{c}, \mathrm{d})$ versions. 


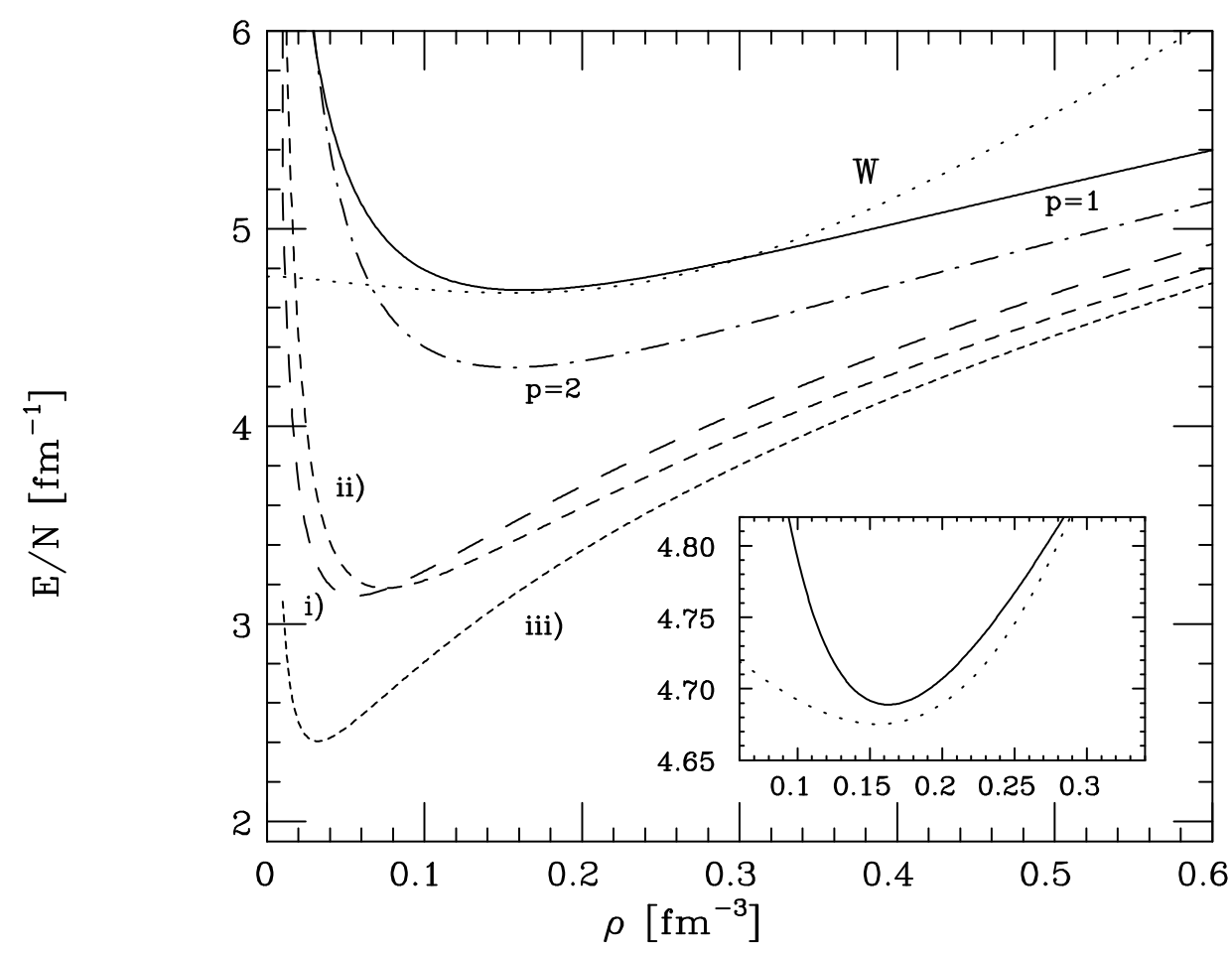

FIG. 2. EOS of QM and EOS of nuclear matter in the Walecka model (dotted line). We show the EOS of QM in CDM for the DM version of the model (dashed lines i) ii) iii) ) and for the SM version with $\mathrm{p}=1$ (solid line) and $\mathrm{p}=2$ (dot-dashed line) . 


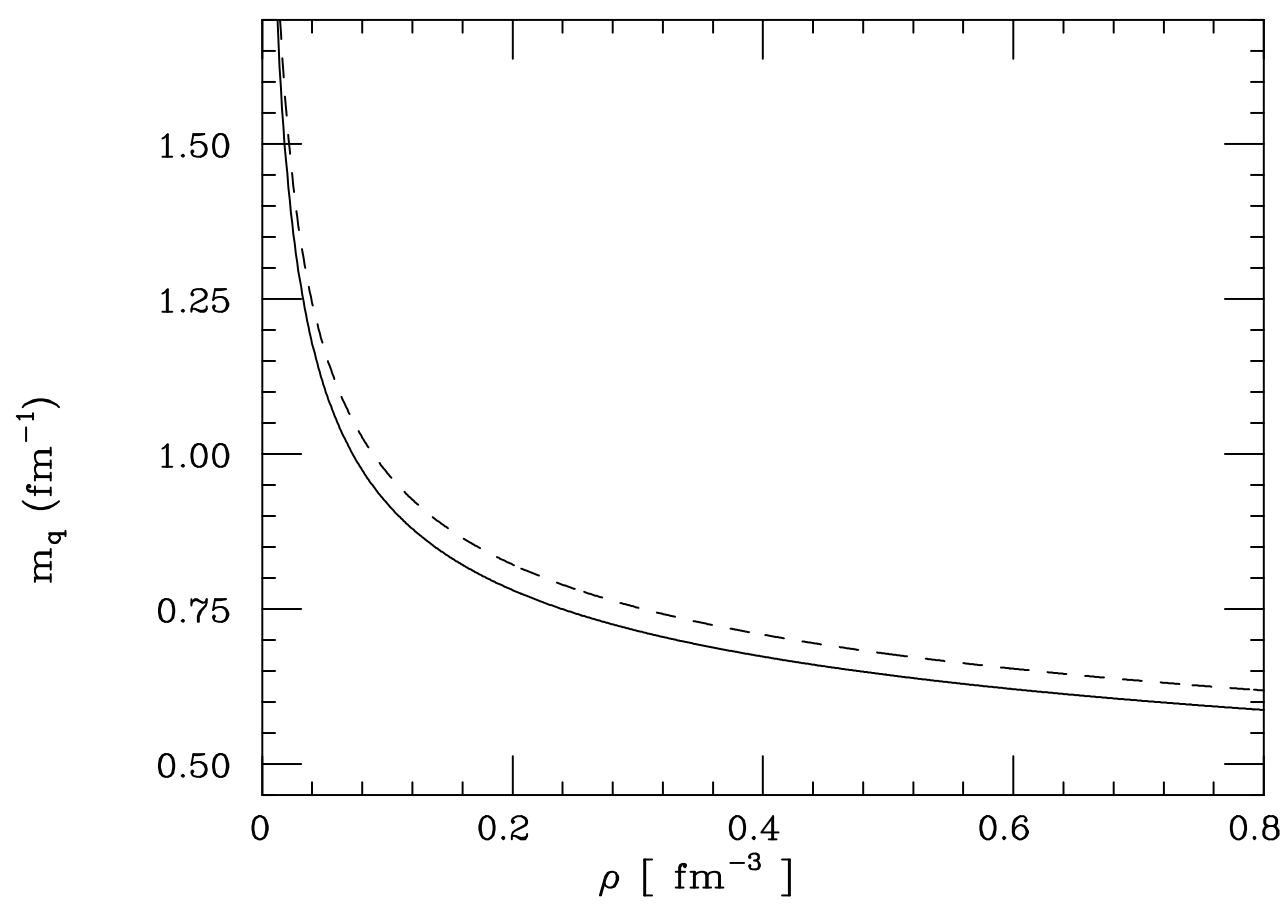

FIG. 3. Effective quark mass versus density: .... . 
a)

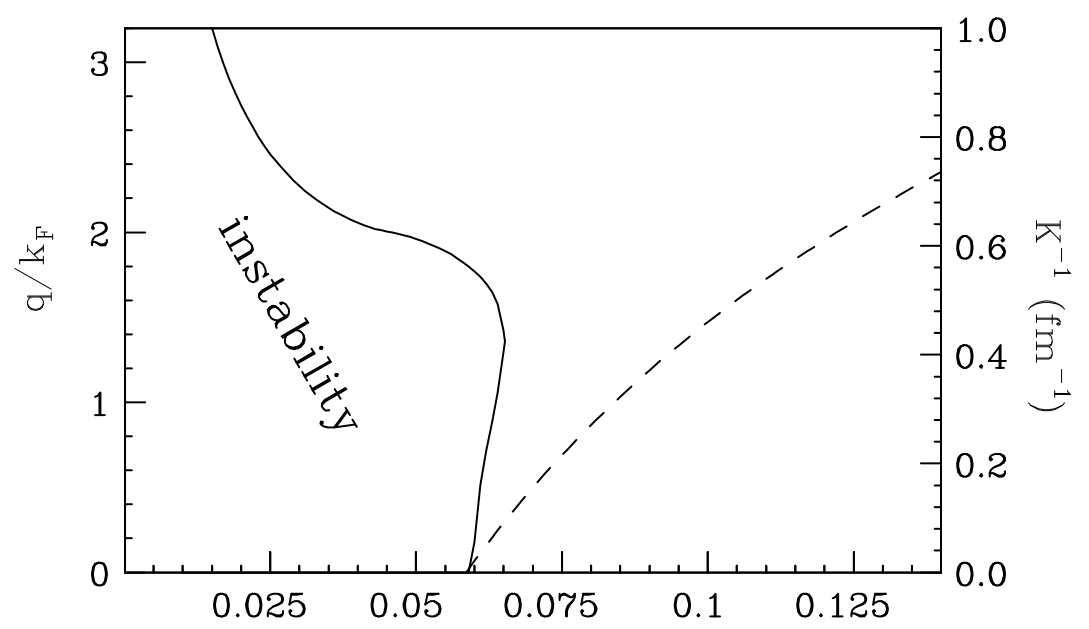

b)

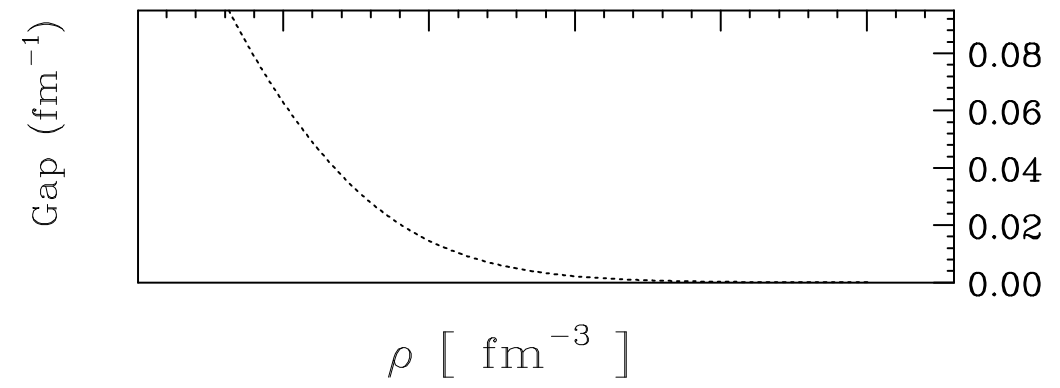

FIG. 4. Compressibility (dashed line), instability region (solid line), and gap (dotted line) for the QM....... . 

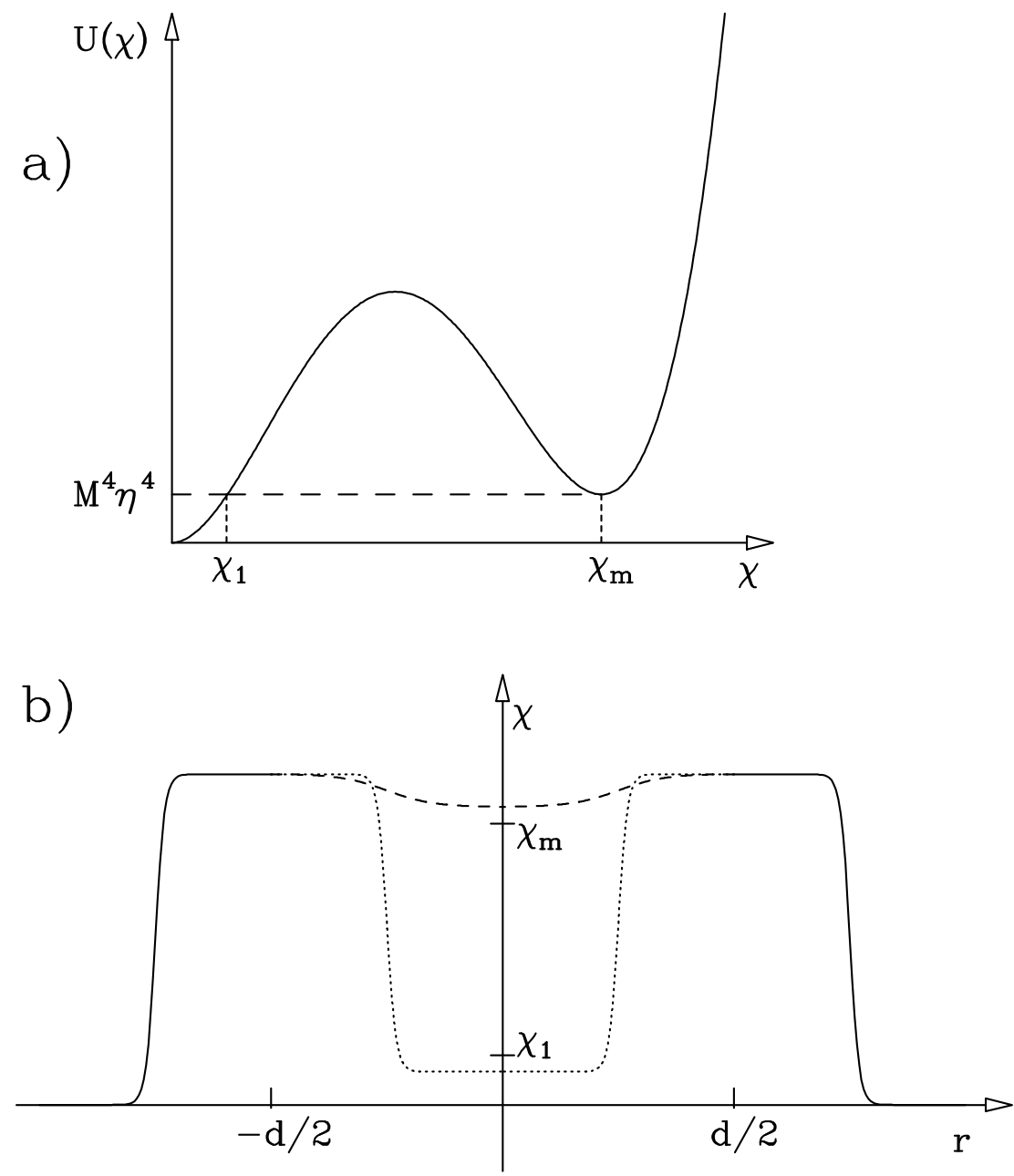

FIG. 5. Behaviour of $\chi$ field in the DM version of the CDM. Potential $U(\chi)$ (a) and shape of $\chi$ field for internucleonic distance $d$. 